\title{
Effect of glycated LDL on microvascular tone in mice: a comparative study with LDL modified in vitro or isolated from diabetic patients
}

\author{
P. Nivoit ${ }^{1}$, N. Wiernsperger ${ }^{1}$, P. Moulin ${ }^{3}$, M. Lagarde ${ }^{2}$, C. Renaudin ${ }^{1}$ \\ ${ }^{1}$ Diabetic Microangiopathy Research Unit, MERCK Santé-INSERM INSA-Lyon, Villeurbanne, Cedex, France \\ 2 INSERM INSA-Lyon, Villeurbanne, France \\ ${ }^{3}$ Department of Endocrinology and Metabolic Diseases, Cardiologic Hospital, Lyon, France
}

\begin{abstract}
Aims/hypothesis. In vitro studies have suggested that glycation of LDL might be implicated in diabetic microangiopathy. We therefore investigated the in vivo effects of LDL glycated in vitro on the mouse skeletal muscle arteriolar tone. Since glycation naturally occurs during diabetes, we also tested the effects of LDL isolated from diabetic patients.

Methods. In anaesthetized mice, the spinotrapezius muscle microcirculation was observed, in situ, using the orthogonal polarization spectral imaging technology. The diameter of terminal $(<20 \mu \mathrm{m})$ and small arterioles $(20-40 \mu \mathrm{m})$ was measured before and after a bolus intravenous injection of glycated LDL followed by a continuous perfusion $(115 \mu \mathrm{g} / \mathrm{kg} / \mathrm{min})$.

Results. A slight decrease of terminal and small arterioles diameter $(<10 \%)$ was observed with native LDL and LDL isolated from healthy subjects. In contrast,
\end{abstract}

mildly glycated LDL induced a clear vasoconstriction of arterioles ( $>15 \%)$, which was further increased when highly glycated LDL was perfused $(>22 \%)$. LDL isolated from diabetic patients mimicked the vasoconstriction obtained with in vitro mildly glycated LDL, which underwent similar glycation as those isolated from diabetic patients.

Conclusion/Interpretation. Our results show in vivo that acute perfusion of both types of glycated LDL (artificially or naturally modified), cause major microvascular modification by enhancing arteriolar tone in skeletal muscle. These findings highlight a new role of glycated LDL at the level of microvessels. We suggest that glycation of LDL could contribute to the impaired vascular reactivity observed in diabetes. [Diabetologia (2003) 46:1550-1558]

Keywords Glycated LDL, microcirculation, OPS imaging, skeletal muscle, arteriole, mouse.
Received: 23 May 2003 / Revised: 28 July 2003

Published online: 24 October 2003

(C) Springer-Verlag 2003

Corresponding author: P. Nivoit, Diabetic Microangiopathy Research Unit, MERCK Santé-INSERM UMR 585 INSALyon, Louis Pasteur Bldg., 11 ave. Jean Capelle, 69621 Villeurbanne, Cedex, France

E-mail: pierre.nivoit@insa-lyon.fr

Abbreviations: OPS, orthogonal polarization spectral; ACEi, angiotensin converting enzyme inhibitors; N-LDL, native LDL; mG-LDL, mildly glycated LDL; hG-LDL, highly glycated LDL; H-LDL, LDL isolated from healthy subjects; D1LDL, LDL isolated from Type 1 diabetic patients; D2-LDL, LDL isolated from Type 2 diabetic patients; TBARS, thiobarbituric acid-reactive substances; TNBS, trinitrobenzenesulfonic acid; MDA, malondialdehyde-(bis(dimethyl acetal)).
Microvascular complications are an important cause of morbidity and mortality in diabetes. Chronic hyperglycaemia has been shown to play a key role in the pathogenic process of microvascular complications. This was supported by clinical studies such as the Diabetes Control and Complications Trial [1] and the United Kingdom Prospective Diabetes Study [2], that have highlighted the need for strict control of glycaemia to prevent or delay the risk of microvascular complications in both Type 1 and Type 2 diabetic patients. The mechanisms whereby hyperglycaemia contributes to the development of diabetic microvascular complications are still poorly understood and several hypotheses have been proposed such as the non-enzymatic glycation of proteins. 
Glycation is a non-enzymatic process, whereby glucose reacts with amino groups located on lysine side chains and $\mathrm{N}$-terminal amino acid residues of proteins leading to structural alterations and, consequently, to functional abnormalities. Glycation could modify long-lived structural proteins of connective tissue, such as collagen, laminin, fibronectin or lens cristallins [3]. This reaction also affects short-lived circulating molecules exposed to increased blood glucose concentrations such as albumin [4], immunoglobulin [5], haemoglobin [6], and lipoproteins [7]. Increased glycation of LDL apolipoprotein B, both in vitro and in vivo, was shown in 1981 [8]. Glycation also affects the lipid fraction of LDL [9].

It is well admitted that glycation of LDL is implicated in the development of macrovascular complications in diabetes [10]. Glycation of LDL enhances their atherogenicity by decreasing the LDL receptor affinity $[11,12]$. The resulting increased half life of LDL associated with the direct effect of glycation favours the binding to arterial proteoglycans [13], which in turn increases LDL exposure to oxidative stress. Once glycated, LDL are more sensitive to oxidation than native LDL $[9,14,15]$, enhancing their uptake by resident macrophages. Glycation of LDL also stimulates platelet aggregation [16], a proatherogenic process. All these events lead to accelerated development of atherosclerosis.

Recently, an emerging role of glycated LDL in diabetic microvascular complications has been discovered. Mildly glycated LDL cause injury to retinal capillary endothelial cells and pericytes [17]. In cultured mesangial cells, glycated LDL activate extracellular signal-regulated protein kinases 1 and 2, an early mitogenic signal [18]. However, the possible influence of glycated LDL, in vivo, at the level of microvessels remains unknown. This prompted us to explore the in vivo effects of acute perfusion of LDL, modified in vitro by glucose, on the mouse skeletal microvascular tone. Since the phenomenon of glycation naturally occurs during diabetes, we also aimed at testing the effects of LDL isolated from Type 1 and Type 2 diabetic patients.

\section{Materials and methods}

Animals. Male C57B16/J mice (Charles River, l'Arbresle, France) weighing $26 \pm 1 \mathrm{~g}$ were used for the experiments. The animals were kept under standard conditions (light 07.00-19.00 h; temperature $22 \pm 1^{\circ} \mathrm{C}$; humidity $50 \pm 10 \%$ ), and fed rodent diet and water ad libitum. The study protocol was approved by the animal use committee of the Claude Bernard University of Lyon.

Surgery and spinotrapezius muscle preparation. All surgical and experimental procedures were carried out under constant flow of isoflurane anaesthesia $(2-2.5 \%)$ in oxygen-nitrous oxide gas mixture $(40 \%-60 \%)$. Throughout the whole experiment, body temperature was kept at $37 \pm 1^{\circ} \mathrm{C}$ by placing the mouse in a supine position on a complete homeothermic blanket system (Harvard Apparatus, Les Ulis, France). Polyethylene catheters (PE-10 intravascular portion fused to PE-50 extravascular portion; Intramedic, FLD, Chilly Mazarin, France) were inserted into the jugular vein for drug administration and into the carotid artery for blood pressure measurement. The rat spinotrapezius muscle preparation [19] was adapted and simplified for use in mice [20]. Briefly, a longitudinal slit was carried out in the skin along the spine from the cervical to the midlumbar region. With iridectomy scissors, the connective tissue that covers the muscle was carefully removed. During the preparation and experimental observations, the skeletal tissue was continuously superfused with a bicarbonate/HEPESbuffered saline containing (in mmol/l): $110 \mathrm{NaCl}, 4.7 \mathrm{KCl}$, $2 \mathrm{CaCl}_{2}, 1.2 \mathrm{MgSO}_{4}, 18 \mathrm{NaHCO}_{3}, 15.39 \mathrm{HEPES}$ and 14.61 HEPES $\mathrm{Na}^{+}$-salt. The temperature of the solution was kept at $36^{\circ} \mathrm{C}$ and the $\mathrm{pH}$ was set at 7.4 by bubbling the solution with $5 \% \mathrm{CO}_{2}$ in $95 \% \mathrm{~N} 2$. Superfusion flow rate was maintained at $4-5 \mathrm{ml} / \mathrm{min}$.

OPS imaging technology. Microcirculation of the mouse spinotrapezius muscle was examined by using a new method based on the Orthogonal Polarization Spectral (OPS) imaging technology [21]. Briefly, the technique eliminates directly reflected green polarized light from an organ surface using an orthogonal placed analyser, which results in clear intravital images of red blood cells flowing through the microcirculation. For our study, we used the OPS imaging Cytoscan Video Microscope (Cytometric, Philadelphia, Pa., USA). Using the S-video out port, the images of the microvessels were examined by a closecircuit video system including a S-VHS video recorder (AG7350, Panasonic, France) and a black and white video monitor (PVM-145 E, Sony, France). This equipment, with the 5X OPS imaging probe positioned on the top of the muscle, allowed the observation of microcirculatory fields of $1.25 \times 0.94 \mathrm{~mm}$. Images were captured and recorded on S-VHS videotape and analysed off-line.

Image analysis. The images were analysed during a playback analysis of the video record using a self-developed image processing system. This system uses pixel size to make microvascular measurements. The system is first calibrated to determine the pixel size using a recording of a micrometer (Zeiss, Le Pecque, France) in both horizontal and vertical orientation. The video signal was digitized through a high-quality monochrome image acquisition board (IMAQ PCI-1407, National Instruments, Le Blanc Mesnil, France) installed in a microcomputer. The image was coded as 768 by 572 pixels, with 256 grey levels. The isotropic orientation of the vessel was used to increase the signal/noise ratio, by integration of the segments perpendicular to the operator's defined segment. The diameters were computed at $50 \%$ of the grey level amplitude of the integrated signal. Arteriolar diameters were determined by measurements of the red blood cell column diameters, the median value of at least five consecutive diameter measurements being considered as the real diameter.

Blood pressure measurements. The arterial blood pressure signal, transmitted to a blood pressure analyser (AH 60-3003, Harvard Apparatus, Les Ulis, France), was digitized at 1000 samples per second. Each second, mean blood pressure and heart rate were computed, displayed and stored using dedicated software IOX (EMKA Technology, Paris, France).

$L D L$ preparation. LDL was prepared from plasma of overnight fasting healthy subjects $(n=15$, smokers $2 / 15)$, Type $1 \quad(n=8$; smokers $2 / 8$; insulin therapy; medication for hypertension 
Table 1. Characteristics of subjects from whom LDLs were obtained

\begin{tabular}{llcl}
\hline Parameter & $\begin{array}{l}\text { Healthy subjects } \\
(n=8)\end{array}$ & $\begin{array}{l}\text { Type 1 diabetic subjects } \\
(n=8)\end{array}$ & $\begin{array}{l}\text { Type 2 diabetic subjects } \\
(n=8)\end{array}$ \\
\hline HbA $_{1 \mathrm{c}}$ & $5.0 \pm 0.2$ & $7.5 \pm 0.6^{\mathrm{a}}$ & $6.9 \pm 0.5^{\mathrm{a}}$ \\
Plasma glucose (mmol/l) & $4.8 \pm 0.1$ & $10.4 \pm 1.2^{\mathrm{a}, \mathrm{b}}$ & $7.5 \pm 0.6^{\mathrm{a}}$ \\
Total cholesterol (mmol/l) & $5.3 \pm 0.3$ & $5.9 \pm 0.3$ & $5.1 \pm 0.4$ \\
HDL chlesterol (mmol/l) & $1.6 \pm 0.1$ & $1.7 \pm 0.1$ & $1.4 \pm 0.1$ \\
Triglycerides (mmol/l) & $1.0 \pm 0.1$ & $1.2 \pm 0.2$ & $1.4 \pm 0.2$ \\
LDL cholesterol (mmol/l) & $3.4 \pm 0.3$ & $3.6 \pm 0.3$ & $3.3 \pm 0.3$ \\
ApoB (g/l) & $1.0 \pm 0.1$ & $1.1 \pm 0.1$ & $1.1 \pm 0.1$ \\
Cholesterol/ApoB (weight/weight) & $1.35 \pm 0.09$ & $1.25 \pm 0.04$ & $1.13 \pm 0.05^{\mathrm{a}}$ \\
\hline
\end{tabular}

Data are means \pm SEM

${ }^{\mathrm{a}} p<0.05$ vs healthy subjects, ${ }^{\mathrm{b}} p<0.05$ vs Type 2 diabetic patients

ACEi 3/8, calcium-channel blockers $1 / 8, \beta$-blockers $2 / 8$; lipidlowering drugs $3 / 8)$ and Type 2 diabetic patients $(n=8$; non smokers; biguanide therapy $5 / 8$, biguanide in association with sulfonylurea or insulin 3/8; medication for hypertension, ACEi $2 / 8$, calcium-channel blockers $2 / 8, \beta$-blocker $2 / 8$; lipid-lowering drugs $4 / 8$ ), mean age $56 \pm 4$ years. Additional characteristics of donors from whom LDL were obtained are shown in Table 1. Blood was collected at the local blood bank from healthy donors and was drawn on CPD (19.6 mmol/l citric acid, $89.4 \mathrm{mmol} / \mathrm{l}$ sodium citrate, $16.1 \mathrm{mmol} / \mathrm{l} \mathrm{NaH}_{2} \mathrm{PO}_{4}$, $128.7 \mathrm{mmol} / \mathrm{l}$ dextrose, $\mathrm{pH}$ 5.6). During medical check up, blood samples were collected on EDTA from diabetic patients at the Department of Endocrinology and Metabolic Diseases and from healthy donors at the Preventive Medicine Institute. Written informed consent was obtained from all donors.

LDL was isolated by sequential ultracentrifugation (400000 g, 6 h) using $\mathrm{NaCl} / \mathrm{KBr}$ density gradient. Ethylenediaminetetraacetic acid (EDTA, $4.2 \mathrm{mmol} / \mathrm{l}$ final) was added to plasma before separation to avoid oxidation. Isolated LDL was

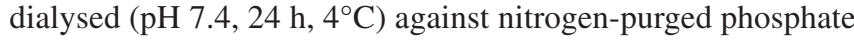
buffered saline (PBS), $270 \mu \mathrm{mol} / \mathrm{l}$ EDTA $(0.01 \% \mathrm{wt} / \mathrm{vol})$ in the dark.

LDL modification. In vitro glycation of LDL was carried out [17]. Briefly, LDL was incubated under nitrogen in presence of $50 \mathrm{mmol} / \mathrm{l} \mathrm{D}$-glucose for 3 days (mildly glycated LDL, mGLDL) or 6 days (highly glycated LDL, hG-LDL) with $200 \mathrm{mmol} / 1$ sodium cyanoborohydride at $37^{\circ} \mathrm{C}$ in $\mathrm{PBS}$, pH 7.4. Native LDL (N-LDL) were incubated without glucose. For NLDL, mG-LDL and hG-LDL, $270 \mu \mathrm{mol} / \mathrm{l}$ EDTA and $1 \mathrm{mmol} / \mathrm{l}$ diethylenetriaminepenta-acetic acid (DTPA) was also added to maintain antioxidant conditions. Modification was finished by an extensive dialysis as described above. LDL preparations were sterile filtered $(0.22 \mu \mathrm{m})$, stored in the dark under nitrogen at $4^{\circ} \mathrm{C}$.

LDL characterization. Determination of purity and alteration in negative charge in LDL preparations was carried out by an agarose gel electrophoresis (Titan Gel Agarose Electrophoresis Systems-Lipoprotein Kit, Helena Laboratories, Saint-Leu la Forêt, France). The relative electrophoretic mobility of each sample was evaluated by measuring the distance in millimetres from the origin to the middle of the sudan black stained band and was expressed as a ratio of the sample to that of the control, N-LDL or freshly LDL isolated from healthy subjects (Fig. 1).

The extent of lipid peroxidation of LDL was estimated by measuring the amount of thiobarbituric acid-reactive substances (TBARS) produced using a colorimetric assay for ma-

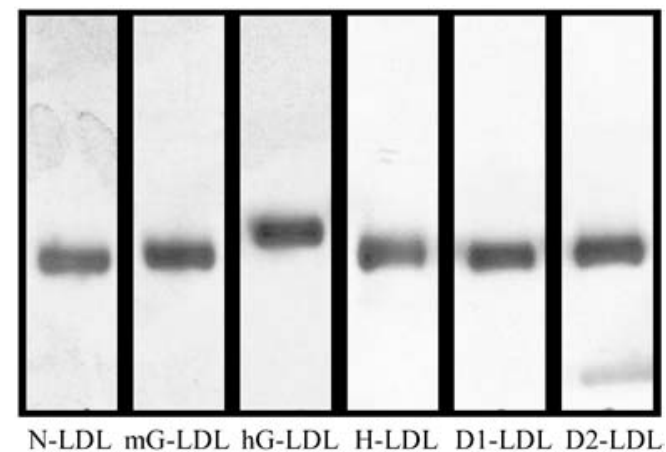

Fig. 1. Representative agarose gel electrophoresis showing electrophoretic mobility of N-LDL, mG-LDL, hG-LDL, H-LDL, D1-LDL and D2-LDL. The mean value of N-LDL and $\mathrm{H}-\mathrm{LDL}$ distance migrated from the origin was $5 \mathrm{~mm}$

londialdehyde [22]. Briefly, $1 \mathrm{ml}$ of $20 \%$ trichloroacetic acid was added to each sample (500 $\mu$ g LDL protein), followed by $1 \mathrm{ml} 0.75 \%$ thiobarbituric acid. The mixture was incubated at $95^{\circ} \mathrm{C}$ for $30 \mathrm{~min}$, cooled centrifuged (3000 rpm, $10 \mathrm{~min}$ ), and assayed by absorption spectroscopy at $550 \mathrm{~nm}$. Freshly diluted malondialdehyde-(bis(dimethyl acetal)) (MDA) was used as standard and results were expressed as nanomoles of MDA equivalents per milligram of protein. Protein concentration of LDL was determined by the BCA protein assay (BCA Protein Assay Kit, Perbio Science France, Bezons, France).

Measurement of the degree of LDL glycation was done by the trinitrobenzenesulfonic acid (TNBS) assay for free lysine and $\mathrm{NH}_{2}$-terminal amino acid residues $[23,24]$. The TNBS assay measures the extent of LDL glycation indirectly because TNBS reacts specifically with free lysines and $\mathrm{NH}_{2}$-terminal amino acid residues to form trinitrophenyl derivatives. The relative reduction of absorbance of $\mathrm{mG}-\mathrm{LDL}$ or hG-LDL compared with N-LDL is a linear function of the concentration of trinitrophenyl derivatives [23]. LDL $(200 \mu \mathrm{l})$ was mixed with $200 \mu \mathrm{l}$ of $4 \%$ NaHCO3, $\mathrm{pH} 8.4$, and $200 \mu \mathrm{l}$ of $0.1 \%$ TNBS. The mixture was incubated in the dark $\left(2 \mathrm{~h}, 37^{\circ} \mathrm{C}\right)$. Optical density was measured at $340 \mathrm{~nm}$. The degree of glycation was calculated from the reduction of absorbance compared with N-LDL. In case of LDL isolated from diabetic subjects, the degree of glycation was calculated from the reduction of absorbance compared with LDL isolated from healthy subjects.

8 -isoprostane in LDL preparation was quantitated using a commercial enzyme immunoassay kit (8-isoprostane EIA kit, Cayman Chemical, Ann Arbor, Mich., USA). 
Experimental protocol. Microvascular studies were conducted on 12-week-old mice. A 45-min stabilization period was allowed after surgery. Four regions of interest within the spinotrapezius muscle that contained terminal arterioles $(<20 \mu \mathrm{m})$ and small arterioles $(20-40 \mu \mathrm{m})$ were selected. For measurement, the four microcirculatory fields were recorded for approx. $60 \mathrm{~s}$ each; the value of arteriolar diameter at this time will be considered as basal diameter. Mice were then allocated into protocol $\mathrm{A}, \mathrm{B}$ or $\mathrm{C}$ in order to test the effect of different types of LDL on skeletal microvascular tone. Protocol A involved perfusion of isotonic saline, the vehicle used to equilibrate the LDL concentration $(n=8)$. Protocol B (LDL modified in vitro) involved perfusion of native LDL (N-LDL, $n=15)$, mildly glycated LDL (mG-LDL, $n=11$ ) or highly glycated LDL (hG-LDL, $n=5$ ). Protocol C (LDL from diabetic patients) involved perfusion of LDL isolated from healthy patients (H-LDL, $n=8)$, Type 1 diabetic patients (D1-LDL, $n=8$ ) or Type 2 diabetic patients (D2-LDL, $n=8$ ). At time 0 , in each protocol, the animal received a bolus intravenous injection of LDL ( $5 \mathrm{mg} / \mathrm{kg}$ body weight, $1 \mathrm{~min}$ ) followed by a slow perfusion of LDL $(115 \mu \mathrm{g} / \mathrm{kg} / \mathrm{min}$ during $1 \mathrm{~h})$. Volumes were matched between all three protocols. Video recording was done at 10, 20, 30, 40, 50 and $60 \mathrm{~min}$ after lipoprotein injection for later off-line analysis of arteriolar diameter.

Statistical analysis. Results are expressed as means \pm SEM. Comparisons between groups of patients were done by $\mathrm{AN}$ OVA followed by the Student-Newman-Keuls'post hoc pairwise test. Between-group comparisons of mice metabolic parameters were performed using the Student-Newman-Keuls test. The data for arteriolar diameter were analysed using twoway analysis of variance to identify time and drug effects. When a significant value was shown, one-way ANOVA was used, followed by the Tukey'post hoc analysis, to determine the time effect in each experimental group and drug effect for each series of experiment. A $p$ value of 0.05 or less was considered statistically significant. Data were analysed using the statistical software SPSS (SPSS France, Paris, France).

\section{Results}

Basal physiologic parameters of C57B16/J mice were similar in all groups: mean arterial blood pressure was $94 \pm 1 \mathrm{mmHg}$, heart rate was $549 \pm 3 \mathrm{bpm}$, terminal arteriole diameter established at $14.9 \pm 0.2 \mu \mathrm{m}$ and small arteriole diameter at $31.3 \pm 0.4 \mu \mathrm{m}$.

\section{Effect of isotonic saline (Protocol A)}

In saline-treated mice, the diameter of terminal and small arterioles remained stable throughout the whole experiment (Fig. 2, Fig. 3). These data show the stability of the mouse spinotrapezius muscle microvascular preparation within the time allowed for intravenous lipoprotein perfusion.

\section{Effects of in vitro modified LDL (Protocol B)}

LDL characterization. Agarose gel electrophoresis of LDL preparations showed a single band showing a

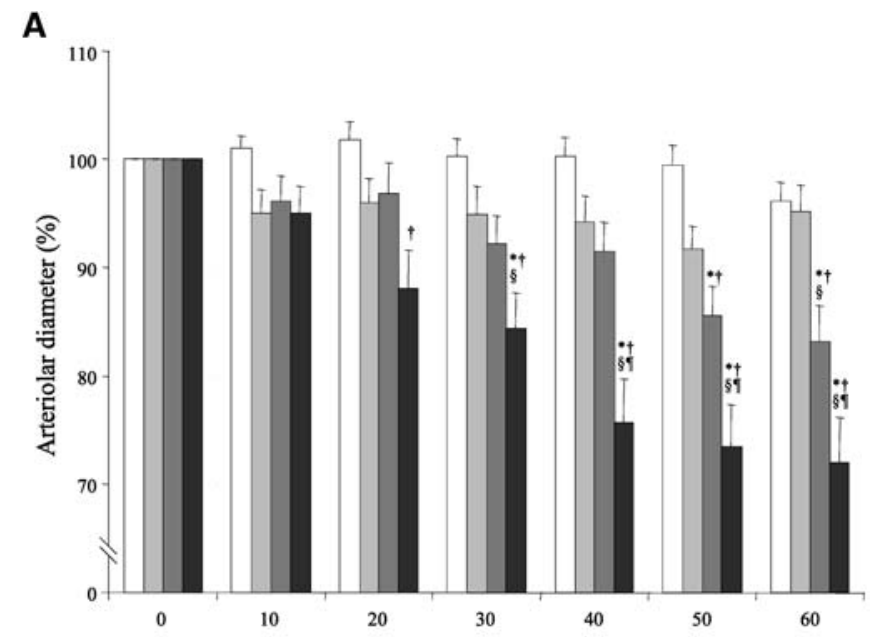

B

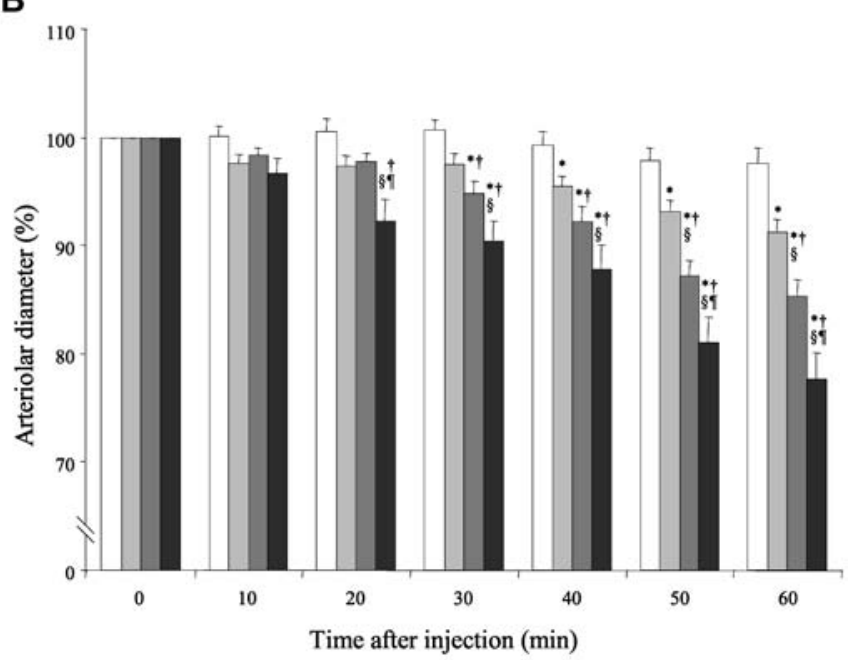

Fig. 2A, B. Effect of intravenous perfusion of N-LDL (light grey bar), mG-LDL (dark grey bar), hG-LDL (black bar) or isotonic saline (white bar) on terminal (A) and small $(\mathbf{B})$ arteriolar diameter in the spinotrapezius muscle of 12 -week-oldmice. Data are means \pm SEM. ${ }^{*} p<0.05$ vs time $0,^{\dagger} p<0.05$ vs saline, ${ }^{\S} p<0.05$ vs N-LDL, "If $p<0.05$ vs mG-LDL

lack of contamination by other lipoprotein subclasses (Fig. 1). The electrophoretic mobility of mG-LDL did not differ from N-LDL (Table 2). In contrast, the electrophoretic mobility of hG-LDL was increased compared to N-LDL and $\mathrm{mG}-\mathrm{LDL}$. The increase in relative mobility of hG-LDL was not due to oxidation because TBARS contents were low and similar in the three types of LDL preparations (Table 2). These data show that the experimental protocol used to prepare in vitro glycated LDL did not modify the oxidative level of lipoproteins. Indeed, incubation of LDL with glucose for 3 days induced a lysine residue derivatisation degree of $15 \%$ in $\mathrm{mG}-\mathrm{LDL}$. This percentage was strongly enhanced to $64 \%$ when the incubation time was doubled ( 6 days in hG-LDL compared to 3 days in $\mathrm{mG}-\mathrm{LDL}$ ) and done in presence of a reducing agent (Table 2). 


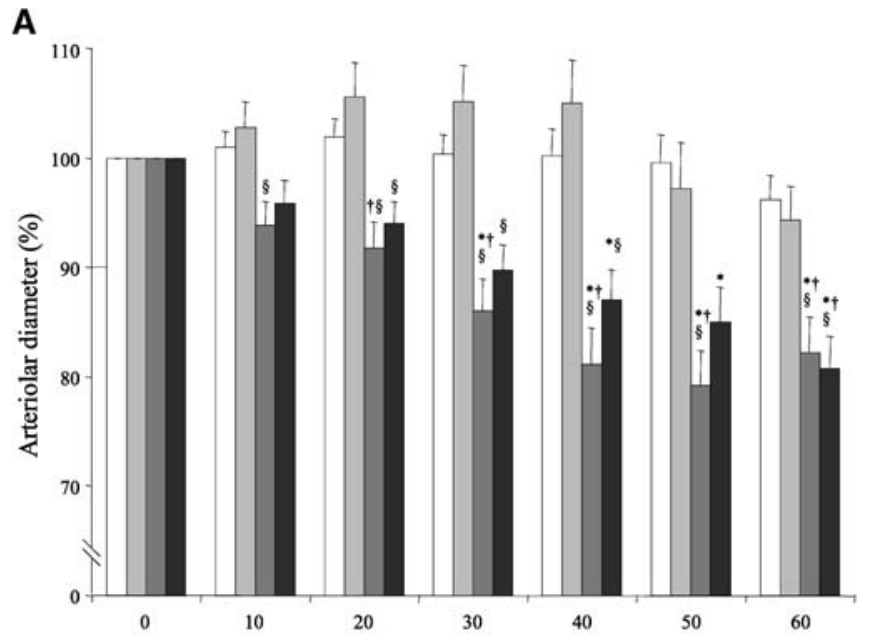

B

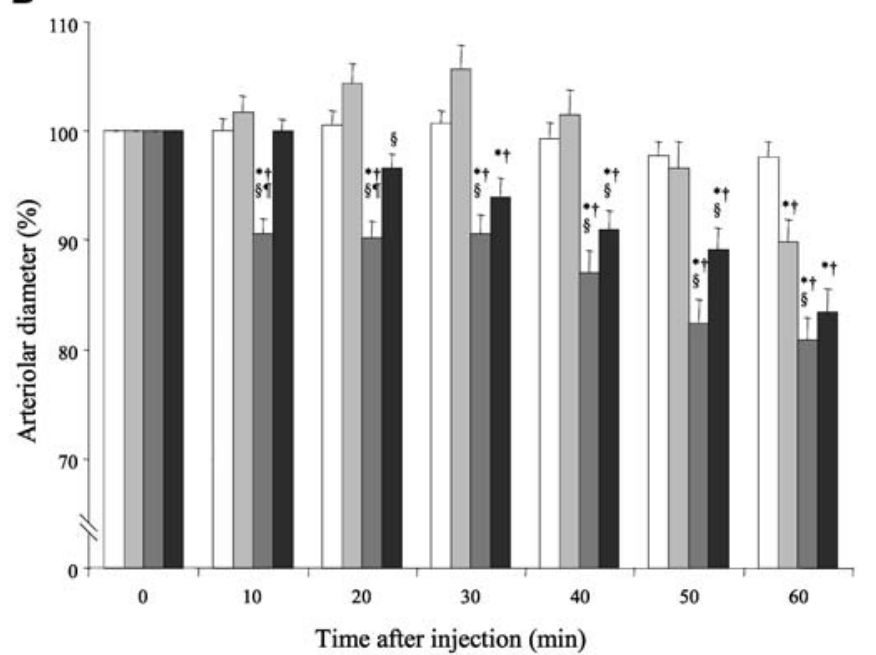

Fig. 3A, B. Effect of intravenous perfusion of H-LDL (light grey bar), D1-LDL (dark grey bar), D2-LDL (black bar) or isotonic saline (white bar) on terminal (A) and small (B) arteriolar diameter in the spinotrapezius muscle of 12-week-oldmice. Data are means \pm SEM. ${ }^{*} p<0.05$ vs time $0,{ }^{\dagger} p<0.05$ vs saline, ${ }^{\S} p<0.05$ vs H-LDL, II $p<0.05$ vs D2-LDL

Effects of intravenous $L D L$ perfusion on arteriolar diameters. N-LDL induced a very modest decrease (less than $10 \%)$ in arteriole diameter, which became significant $(\mathrm{p}<0.05)$ for small arterioles only at time $40 \mathrm{~min}$
(Fig. 2B). However, no difference was observed compared to saline group at any time. In contrast, a clear vasoconstriction was obtained (more than 10\%) in both terminal and small arterioles in mG-LDL-treated mice. The effect appeared earlier in small arterioles (time $30 \mathrm{~min}$, Fig. 2B) than in terminal arterioles (time $50 \mathrm{~min}$, Fig. 2A). At the end of the experiment, with mG-LDL, a vasoconstriction of $17 \%$ and $15 \%$ was obtained for terminal and small arterioles respectively. In hG-LDL-treated mice, the vasoconstrictor effect was even more pronounced since it appeared very early (time $20 \mathrm{~min}$ ) and its amplitude was greater $(>20 \%)$ than in mG-LDL. At the end of the experiment, in hG-LDL-perfused mice, vasoconstriction was $28 \%$ and $23 \%$ for terminal and small arterioles, respectively.

Effects of LDL isolated from diabetic patients (Protocol C)

Characteristics of diabetic donors. The blood glucose concentration was increased in Type 1 diabetic patients (2.2-fold) and in Type 2 diabetic patients (1.6fold) compared to healthy subjects. The percentage of $\mathrm{HbA}_{1 \mathrm{c}}$ was around 7\% in diabetic patients. Cholesterol, triglycerides, HDL, LDL and Apo B were similar in all three groups. Cholesterol to protein weight ratio was lower in LDL from diabetic patients compared to the healthy subjects, reaching statistical significance only for Type 2 diabetic patients (Table 1).

LDL characterization. The purity of the LDL preparations was shown by a single band on the agarose gel electrophoresis (Fig. 1). D1-LDL and D2-LDL isolated from diabetic subjects showed no change in electrophoretic mobility on agarose gel compared to $\mathrm{H}$ LDL isolated from healthy subjects as well as N-LDL and $\mathrm{mG}-\mathrm{LDL}$ (Table 2). The TBARS contents of $\mathrm{H}-$ LDL, D1-LDL and D2-LDL were similar and comparable to values obtained for N-LDL, mG-LDL and hG-LDL (Table 2). The degree of derivatisation of lysine residues was similar between D1-LDL and D2-

Table 2. Degree of modification of LDL preparations

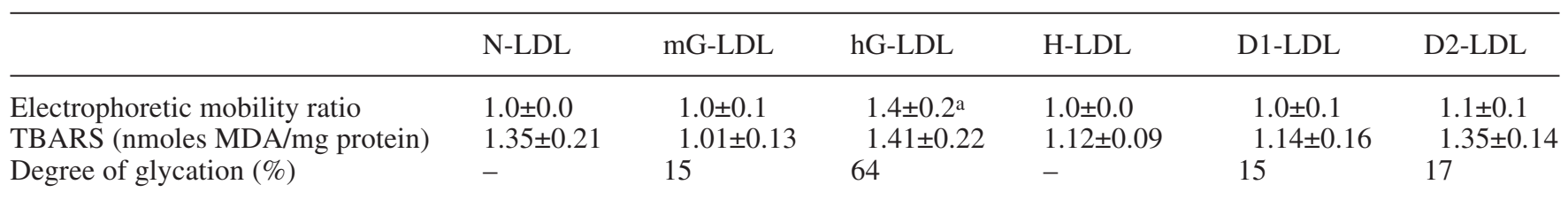

The electrophoretic mobility of in vitro glycated LDL (mGLDL, hG-LDL) and LDL isolated from diabetic subjects (D1LDL, D2-LDL) is expressed as the ratio to their own controls: N-LDL or H-LDL. TBARS contents are expressed in nmol $\mathrm{MDA} / \mathrm{mg}$ protein. The degree of glycation is expressed as the percentage of relative reduction of absorbance of in vitro glycated LDL (mG-LDL, hG-LDL) and LDL isolated from diabetic subjects (D1-LDL, D2-LDL) compared to their own control: N-LDL or H-LDL

Data are means \pm SEM

a $p<0.05$ vs controls 
LDL ( $15 \%$ and $17 \%$, respectively) compared to $\mathrm{H}-$ LDL (Table 2). This percentage of glycation was equal to that obtained with LDL mildly modified in vitro by glucose (mG-LDL: $15 \%$ ).

Effects of intravenous $L D L$ perfusion on arteriolar diameters. H-LDL perfusion induced a slight oscillation of arteriolar diameter (less than 10\%), which reached statistical significance in small arterioles only at time $60 \mathrm{~min}$ (Fig. 3B). On the contrary, a pronounced vasoconstrictor effect was observed in both terminal and small arterioles in D1-LDL- and D2LDL-perfused mice. The effect started at time $10 \mathrm{~min}$ for both terminal and small arterioles with D1-LDL. At the end of the experiment, the extent of D1-LDLinduced vasoconstriction reached the value of $18 \%$ for terminal arterioles and 19\% for small arterioles. With D2-LDL, the effect started at time 20 min for both terminal and small arterioles. At the end of the experiment, the D2-LDL-induced vasoconstriction was $19 \%$ for terminal arterioles and $17 \%$ for small arterioles.

\section{Discussion}

The present study showed that, in vivo, glycated LDL affect microvascular tone in the skeletal muscle. The arteriolar vasoconstriction induced by LDL was strongly potentiated by increasing their degree of glycation in vitro. These results were validated and strengthened by concordant results obtained with human LDL naturally modified during diabetes. This novel result adds an important in vivo perspective to data already reported by others about the possible implication of glycated LDL in the development of diabetic microcomplications $[17,18]$.

We have visualized the skeletal muscle microcirculation of the mouse by using a novel system, the OPS imaging technology [21]. Using haemoglobin absorption to visualize the red blood cells, OPS imaging produces highly contrasted images of microvascular networks without the use of fluorescent dyes for contrast enhancement. This allows observation of the microcirculation on the surface of solid organs. This new technology provides a useful alternative for intravital microscopy when transillumination cannot be carried out due to insufficient length of muscle to be exteriorized. We previously reported that this technique allowed us to observe in vivo the mouse spinotrapezius muscle microcirculation and gave valid quantitative measurements of the microvascular diameter [25].

During this study, we chose to explore two different types of glycated LDL, one that was artificially glycated and another that was naturally glycated in vivo. We studied the impact of LDL modified in vitro using the protocol described in another study [17] because the mildly glycated LDL obtained have a degree of glycation similar to that occurring in diabetic patients with poor glycaemic control. Moreover by adding a reducing agent, we amplified the in vitro glycation of LDL in order to obtain glycated particles with high degree of modifications. Based on TNBS assay, approximately $15 \%$ of lysine residues of $\mathrm{mG}-\mathrm{LDL}$ and $65 \%$ of lysine residues of hG-LDL were modified by glycation compared to N-LDL. In these conditions we visualized in vivo that LDL acts on microvascular tone by decreasing diameter of both small and terminal arterioles. This effect was obtained with a physiological increase of LDL. Indeed using our lipoprotein perfusion protocol in preliminary studies (data not shown), we maintained a constant dose of circulating LDL without changes of the clearance rate between mildly glycated LDL and native LDL during the time allowed for the experiment. Assuming that the circulating blood volume of mice is approximately $2 \mathrm{ml}$ [26], the injected dose of glycated LDL therefore seems to be clinically relevant in the range of the serum glycated LDL concentration measured in diabetic patients [27]. With more severe LDL glycation, the vasoconstrictor effect was strongly potentiated from $15 \%$ to $22 \%$ at the end of the experiments. This increase of microvascular tone was not due to differences in the lipid composition of lipoproteins since NLDL, mG-LDL and hG-LDL were prepared with plasma obtained from the same donors.

In order to test the relevance of our findings with artificially glycated LDL and since glycation is enhanced in Type $1[28,29]$ as well as in Type 2 diabetes [27, 30,31 , we decided to investigate the effects of LDL isolated from diabetic patients. In our study, the diabetic patients were hyperglycaemic and had higher glycosylated haemoglobin compared to healthy subjects. In the literature, the degree of LDL glycation in diabetes is correlated with short and medium term indicators of glycaemic control. For example, it has been shown that in Type 1 diabetic patients, LDL glycation was correlated with mean plasma glucose, plasma protein glycation and glycosylated haemoglobin $\left(\mathrm{HbA}_{1 \mathrm{c}}\right)$ and that increased LDL glycation was present even in normolipidaemic diabetic patients who had reasonable glycaemic control [28]. In the same way, in Type 2 diabetic hyperlipidaemic patients, LDL glycation was correlated with mean plasma glucose and fructosamine [27]. We showed here that LDL isolated from Type 1 or Type 2 diabetic patients induced a clear increased microvascular tone whereas those isolated from healthy subjects did not. LDL isolated from diabetic patients mimicked the vasoconstriction obtained with in vitro mG-LDL which had the same amount of glycation than those isolated from diabetic patients. Taken together, these data show that glycation, per se, modifies the function of LDL, which become a strong active factor able to affect microvascular tone.

The mechanisms underlying this increased microvascular tone remain undefined. Several hypotheses 
can be made. Firstly, glycation of LDL decreases their affinity for the LDL receptor resulting in a decrease of the catabolism of glycated LDL [11]. However, we did not observe modifications of the in vivo plasma clearance rate of mildly glycated LDL compared to native LDL during the LDL perfusion time (data not shown) suggesting that glycation acts by another mechanism in our experiments.

Secondly, glycated LDL could exert this vasomotor effect via glycoxidation. In vitro and in vivo glycated LDL are prone to oxidative modifications $[9,14,15]$ that could affect the vasomotor response of isolated macro and microvessels [32]. However, no increased arteriolar vasoconstriction was observed with glycoxidized LDL compared to mildly glycated LDL in our preliminary study done in vivo (data not shown); failure to find a more pronounced effect with glycoxidized LDL could be explained by the different time course of the LDL clearance due to oxidation [33], leading to a very rapid and strong diminution of the circulating concentration of glycoxidized LDL. Therefore, the oxidative process cannot explain the vasoconstriction observed with glycated LDL in our study, supported by the fact that TBARS concentration remained low.

Thirdly, a qualitative modification found in LDL isolated from Type 1 [34] and Type 2 [35] diabetic patients that might potentially account for this increased microvascular tone is the distribution of LDL in smaller and denser subfractions. Small dense LDL subtypes are characterised by depletion in cholesterol content and a relative enrichment in apo B [36]. In our study, diabetic patients have a low cholesterol to protein weight ratio in the LDL fraction reaching statistical significance only for Type 2 diabetic patients, indicating predominance of small dense LDL, as reported previously [37, 38]. This could potentiate the effect of glycation and explain why the effects of naturally glycated LDL on arteriolar tone appeared more rapidly than those of artificially glycated ones. However similar effects were observed with LDL isolated from Type 1 (normal size) and Type 2 (small size) suggesting that glycation is the more important factor.

Fourthly, LDL preparation could liberate isoprostanes [39], such as 8 -epi-PGF $2 \alpha$ a potent vasoconstrictor. However, in our LDL preparation, done under an antioxidant condition and finished by a dialysis, only a negligible concentration of 8 -iso- $\mathrm{PGF}_{2 \alpha}$ was measured (6-8 pg/mg LDL). Haemodynamic investigations with 8 -epi-PGF $2 \alpha$ have shown vasomotor effects in the higher micromolar range [40].

Finally, the most likely hypothesis to explain the vasoconstricting effect observed is that glycated LDL might modify the balance of vasoactive substances produced by the endothelium by decreasing the synthesis and the bioactivity of nitric oxide [23] and/or increasing the production of vasoconstricting substances such as endothelin-1 [41, 42]. Future studies will be implemented to identify the precise mechanisms.
Whatever the mechanisms involved, by acting on microvascular tone, glycated LDL might contribute to endothelial dysfunction. In diabetes, endothelial dysfunction is considered as a critical and initiating factor in the development of chronic diabetic macro- and microvascular disease and it becomes a great challenge to understand its mechanisms and aetiology [43]. Most of the studies had focused on hyperglycaemia as the main cause of this impaired vascular function but compelling evidence in healthy [44], hypercholesterolaemic $[45,46]$ and diabetic patients [47] suggests that LDL cholesterol concentration could also be a major cause of alteration of the endothelial function. However, in diabetic patients, impairment of endothelial function occurs even under normocholesterolaemic conditions with a normal LDL concentration [48] and intensive lipid lowering therapy by statins failed to improve vascular reactivity in several studies [49]. It has been also shown that LDL isolated from Type 2 diabetic patients produced greater inhibition of endothelium-dependent relaxation to acetylcholine in rabbit thoracic aorta than did equal concentrations of LDL isolated from control subjects [50]. The authors suggested that qualitative abnormalities of diabetic LDL are able to increase their potency in inhibiting endothelium-dependent relaxation. Our results are in agreement with these findings and suggest that long-term elevation and accumulation of glycated LDL could impair regulation of vascular reactivity, contributing to the development of the chronic diabetic microvascular complications.

Acknowledgements. We thank Dr I. Berard from MERCK Santé for her expertise in LDL, Dr E. Michoud for his self-developed image processinf system, Dr V. Pruneta for help in LDL characterization on agarose gel. We also thank Mrs A.M. Chevrier for analytical assay and the nurses from the clinical unit for blood drawing.

\section{References}

1. The Diabetes Control and Complications Trial Research Group (1993) The effect of intensive treatment of diabetes on the development and progression of long-term complications in insulin-dependent diabetes mellitus. N Engl J Med 329:977-986

2. UK Prospective Diabetes Study (UKPDS) Group (1998) Intensive blood-glucose control with sulphonylureas or insulin compared with conventional treatment and risk of complications in patients with type 2 diabetes (UKPDS 33). Lancet 352:837-853

3. Brownlee M (1992) Nonenzymatic glycosylation of macromolecules. Prospects of pharmacologic modulation. Diabetes 41 [Suppl 2]:57-60

4. Kennedy AL, Merimee TJ (1981) Glycosylated serum protein and hemoglobin A1 levels to measure control of glycemia. Ann Intern Med 95:56-58

5. Danze PM, Tarjoman A, Rousseaux J, Fossati P, Dautrevaux M (1987) Evidence for an increased glycation of IgG in diabetic patients. Clin Chim Acta 166:143-153

6. Koenig RJ, Peterson CM, Jones RL, Saudek C, Lehrman M, Cerami A (1976) Correlation of glucose regulation and 
hemoglobin AIc in diabetes mellitus. N Engl J Med 295: 417-420

7. Lyons TJ (1992) Lipoprotein glycation and its metabolic consequences. Diabetes 41 [Suppl 2]:67-73

8. Schleicher E, Deufel T, Wieland OH (1981) Non-enzymatic glycosylation of human serum lipoproteins. Elevated epsilon-lysine glycosylated low density lipoprotein in diabetic patients. FEBS Lett 129:1-4

9. Bucala R, Makita Z, Koschinsky T, Cerami A, Vlassara H (1993) Lipid advanced glycosylation: pathway for lipid oxidation in vivo. Proc Natl Acad Sci USA 90:6434-6438

10. Graier WF, Kostner GM (1997) Glycated low-density lipoprotein and atherogenesis: the missing link between diabetes mellitus and hypercholesterolaemia? Eur J Clin Invest 27:457-459

11. Steinbrecher UP, Witztum JL (1984) Glucosylation of low-density lipoproteins to an extent comparable to that seen in diabetes slows their catabolism. Diabetes 33:130-134

12. Lopes-Virella MF, Sherer GK, Lees AM et al. (1982) Surface binding, internalization and degradation by cultured human fibroblasts of low density lipoproteins isolated from type 1 (insulin-dependent) diabetic patients: changes with metabolic control. Diabetologia 22:430-436

13. Edwards IJ, Wagner JD, Litwak KN, Rudel LL, Cefalu WT (1999) Glycation of plasma low density lipoproteins increases interaction with arterial proteoglycans. Diabetes Res Clin Pract 46:9-18

14. Sobal G, Menzel J, Sinzinger H (2000) Why is glycated LDL more sensitive to oxidation than native LDL? A comparative study. Prostaglandins Leukot Essent Fatty Acids 63:177-186

15. Bowie A, Owens D, Collins P, Johnson A, Tomkin GH (1993) Glycosylated low density lipoprotein is more sensitive to oxidation: implications for the diabetic patient? Atherosclerosis 102:63-67

16. Ferretti G, Rabini RA, Bacchetti T et al. (2002) Glycated low density lipoproteins modify platelet properties: a compositional and functional study. J Clin Endocrinol Metab 87:2180-2184

17. Lyons TJ, Li W, Wells-Knecht MC, Jokl R (1994) Toxicity of mildly modified low-density lipoproteins to cultured retinal capillary endothelial cells and pericytes. Diabetes 43:1090-1095

18. Jenkins AJ, Velarde V, Klein RL et al. (2000) Native and modified LDL activate extracellular signal-regulated kinases in mesangial cells. Diabetes 49:2160-2169

19. Gray SD (1973) Rat spinotrapezius muscle preparation for microscopic observation of the terminal vascular bed. Microvasc Res 5:395-400

20. Nivoit P, Renaudin C, Lagarde M, Wiernsperger N (2001) Mouse spinotrapezius muscle preparation for microscopic study of skeletal muscle vascular resistance bed: prospective studies with ox-LDL. Proceedings of the 7th World Congress for Microcirculation P2-25 (Abstract)

21. Groner W, Winkelman JW, Harris AG et al. (1999) Orthogonal polarization spectral imaging: a new method for study of the microcirculation. Nat Med 5:1209-1212

22. Yagi K (1976) A simple fluorometric assay for lipoperoxide in blood plasma. Biochem Med 15:212-216

23. Posch K, Simecek S, Wascher TC et al. (1999) Glycated low-density lipoprotein attenuates shear stress-induced nitric oxide synthesis by inhibition of shear stress-activated L-arginine uptake in endothelial cells. Diabetes 48: 1331-1337

24. Duell PB, Oram JF, Bierman EL (1990) Nonenzymatic glycosylation of HDL resulting in inhibition of high-affinity binding to cultured human fibroblasts. Diabetes 39: 1257-1263

25. Nivoit P, Renaudin C, Lagarde M, Wiernsperger N (2002) Visualisation of the mouse skeletal muscle microcirculation by OPS imaging system: Validation with vasoactive drugs. Proceedings of the 3rd International Amsterdam Mouse Symposium

26. Diehl KH, Hull R, Morton D et al. (2001) A good practice guide to the administration of substances and removal of blood, including routes and volumes. J Appl Toxicol 21: $15-23$

27. Akanji AO, Abdella N, Mojiminiyi OA (2002) Determinants of glycated LDL levels in nondiabetic and diabetic hyperlipidaemic patients in Kuwait. Clin Chim Acta 317: 171-176

28. Lyons TJ, Baynes JW, Patrick JS, Colwell JA, Lopes-Virella MF (1986) Glycosylation of low density lipoprotein in patients with type 1 (insulin-dependent) diabetes: correlations with other parameters of glycaemic control. Diabetologia 29:685-689

29. Klein RL, Laimins M, Lopes-Virella MF (1995) Isolation, characterization, and metabolism of the glycated and nonglycated subfractions of low-density lipoproteins isolated from type I diabetic patients and nondiabetic subjects. Diabetes 44:1093-1098

30. Moro E, Alessandrini P, Zambon C et al. (1999) Is glycation of low density lipoproteins in patients with Type 2 diabetes mellitus a LDL pre-oxidative condition? Diabet Med 16:663-669

31. Sanguinetti SM, Schreier LE, Elbert A, Fasulo V, Ferrari N, Wikinski RL (1999) Detection of structural alterations in LDL isolated from type 2 diabetic patients: application of the fructosamine assay to evaluate the extent of LDL glycation. Atherosclerosis 143:213-215

32. Cox DA, Cohen ML (1996) Effects of oxidized low-density lipoprotein on vascular contraction and relaxation: clinical and pharmacological implications in atherosclerosis. Pharmacol Rev 48:3-19

33. Nivoit P, Renaudin C, Lagarde M, Wiernsperger N (2002) In vivo microvascular responses to native and oxidized low density lipoprotein in mouse spinotrapezius muscle. Diabetes 51 [Suppl 2] A496 2038-PO (Abstract)

34. Sibley SD, Hokanson JE, Steffes MW et al. (1999) Increased small dense LDL and intermediate-density lipoprotein with albuminuria in type 1 diabetes. Diabetes Care 22: $1165-1170$

35. Haffner SM (2002) Lipoprotein disorders associated with type 2 diabetes mellitus and insulin resistance. Am J Cardiol 90:55-6136

36. Kwiterovich PO Jr (1988) HyperapoB: a pleiotropic phenotype characterized by dense low-density lipoproteins and associated with coronary artery disease. Clin Chem 34:B71-B77

37. Feingold KR, Grunfeld C, Pang M, Doerrler W, Krauss RM (1992) LDL subclass phenotypes and triglyceride metabolism in non-insulin-dependent diabetes. Arterioscler Thromb 12:1496-1502

38. Austin MA, Edwards KL (1996) Small, dense low density lipoproteins, the insulin resistance syndrome and noninsulin-dependent diabetes. Curr Opin Lipidol 7:167-171

39. Sobal G, Menzel EJ, Sinzinger H (2000) The effects of glycation/glycoxidation on the liberation of 8-epi- $\mathrm{PGF}_{2 \alpha}$ from low density lipoprotein during its in vitro oxidation. Prostaglandins Leukot Essent Fatty Acids 62:217-224

40. Michoud E, Lecomte M, Lagarde M, Wiernsperger $N$ (1998) In vivo effect of 8 -epi-PGF $2 \alpha$ on retinal circulation in diabetic and non-diabetic rats. Prostaglandins Leukot Essent Fatty Acids 59:349-355 
41. Romerio SC, Linder L, Flammer J, Haefeli WE (2000) Correlation between apolipoprotein B and endothelin-1-induced vasoconstriction in humans. Peptides 21:871-874

42. Jenkins AJ, Li W, Moller K et al. (1999) Pre-enrichment of modified low density lipoproteins with alpha-tocopherol mitigates adverse effects on cultured retinal capillary cells. Curr Eye Res 19:137-145

43. De Vriese AS, Verbeuren TJ, van d, V, Lameire NH, Vanhoutte PM (2000) Endothelial dysfunction in diabetes. Br J Pharmacol 130:963-974

44. Steinberg HO, Bayazeed B, Hook G, Johnson A, Cronin J, Baron AD (1997) Endothelial dysfunction is associated with cholesterol levels in the high normal range in humans. Circulation 96:3287-3293

45. Kaufmann PA, Gnecchi-Ruscone T, Schafers KP, Luscher TF, Camici PG (2000) Low density lipoprotein cholesterol and coronary microvascular dysfunction in hypercholesterolemia. J Am Coll Cardiol 36:103-109

46. Chowienczyk PJ, Watts GF, Cockcroft JR, Ritter JM (1992) Impaired endothelium-dependent vasodilation of forearm resistance vessels in hypercholesterolaemia. Lancet 340:1430-1432

47. Clarkson P, Celermajer DS, Donald AE et al. (1996) Impaired vascular reactivity in insulin-dependent diabetes mellitus is related to disease duration and low density lipoprotein cholesterol levels. J Am Coll Cardiol 28:573-579

48. McVeigh GE, Brennan GM, Johnston GD et al. (1992) Impaired endothelium-dependent and independent vasodilation in patients with type 2 (non-insulin-dependent) diabetes mellitus. Diabetologia 35:771-776

49. Van Etten RW, Koning EJ de, Honing ML, Stroes ES, Gaillard CA, Rabelink TJ (2002) Intensive lipid lowering by statin therapy does not improve vasoreactivity in patients with type 2 diabetes. Arterioscler Thromb Vasc Biol 22:799-804

50. McNeill KL, Fontana L, Russell-Jones DL, Rajman I, Ritter JM, Chowienczyk PJ (2000) Inhibitory effects of low-density lipoproteins from men with type II diabetes on endothelium-dependent relaxation. J Am Coll Cardiol $35: 1622-1627$ 